\title{
A simple spatial model for self-heating compost piles
}

\author{
H. S. Sidhu ${ }^{1} \quad$ M. I. Nelson ${ }^{2} \quad$ X. D. Chen ${ }^{3}$
}

(Received 16 August 2006; revised 11 March 2007)

\begin{abstract}
We model the increase in temperature in compost piles due to micro-organisms undergoing exothermic reactions. The model incorporates two types of heat release: one due to biological activity; and the other due to the oxidation of cellulosic materials. In this study we also include the consumption of oxygen. We investigate the bifurcation behaviour and compare the results obtained from models that include and exclude oxygen consumption in both one- and two-dimensional geometries.
\end{abstract}

\section{Contents}

See http://anziamj.austms.org.au/ojs/index.php/ANZIAMJ/article/view/86 for this article, (C) Austral. Mathematical Soc. 2007. Published May 20, 2007. ISSN $1446-8735$ 


\section{Mathematical formulation}

3 Results

3.1 One-dimensional case . . . . . . . . . . . . . . . C C140

3.2 Two-dimensional case . . . . . . . . . . . . . . . . . C144

4 Conclusions

5 Nomenclature and typical parameter values

References

C148

\section{Introduction}

The phenomenon of spontaneous ignition due to internal heating in bulk solids such as coal, grain, hay, wool wastes, etc., can be described by thermal explosion theory $[1,5]$. In such models heat release is usually represented by a single Arrhenius reaction and combustion is initiated when heat loss is unable to balance the heat generated by the internal heating of the bulk material. However, in industrial processes involving large volumes of bulk organic materials there are two sources of heat generation: a low temperature process involving the growth and respiration of micro-organisms, such as aerobic mould-fungi and bacteria, and a high temperature process due to oxidation of cellulosic materials. Examples of processes where biological heating is important include large-scale composting operations [12], the storage of industrial waste fuel, such as municipal solid waste, and landfills. In these examples, self-heating due to biological activity is considered desirable, for example in composting [2]. Elevated temperatures of the order $70^{\circ} \mathrm{C}$ to $90^{\circ} \mathrm{C}$ may be found within a few months or even a few days [8]. Although it has been recognised for over twenty years that biological heating may be an indispensable prelude to self-ignition [1, p.373], very little information is available regarding the mechanism of fires when biological self-heating is 
involved, despite fires occurring at landfills worldwide [9].

The case when self-heating is entirely due to cellulosic oxidation is well known $[1,5]$. Nelson et al. [10] analysed a spatially uniform model, based upon the theory of Semenov for thermal explosions, for self-heating in compost piles. They investigated the cases when self-heating is due to purely biological heat release and due to a combination of biological and chemical heat release. Since the system investigated was described by a single (but nonlinear), first order ordinary differential equations, with few parameters, the authors were able to thoroughly investigate the generic steady-state behaviour of the system when parameters were varied by using singularity theory [7]. Recently, Sidhu et al. [14] investigated the spatial behaviour of a two dimensional rectangular slab representing the compost pile when oxygen consumption was neglected. They showed that their model was able to predict both the low as well as elevated temperature branches. Here we extend the model from the latter investigation to include the consumption of oxygen. The work investigated here forms the most realistic system of composting so far. We compare the behaviour of the models (with and without oxygen consumption) for both the one- and two-dimensional geometries. We believe that this preliminary analysis is a constructive step towards an understanding of the self-heating process in industrial size compost piles.

\section{Mathematical formulation}

Here we extend the previous model of Sidhu et al. [14] and introduce oxygen as a variable. Ignoring the depletion of cellulosic materials and biomass, the mathematical model of self-heating in a compost heap reduces to two equations describing the temperature and oxygen distributions within the pile. For simplicity, local thermal equilibrium is assumed which is a common assumption for porous medium and packed particle beds [11]. Thus interphase (solid particles and gas) temperature and concentration gradients 
are ignored and a single temperature and concentration are used to describe local behaviour. We consider an idealized two-dimensional pile which has a domain described by a rectangular slab with length $L$ and height $h$. The two-variable PDEs governing the systems are

$$
\begin{aligned}
& \text { Temperature }(T) \text { for } x \in[0, L] \text { and } y \in[0, h] \\
& \begin{array}{l}
(\rho C)_{\mathrm{eff}} \frac{\partial T}{\partial t}=k_{\mathrm{eff}} \nabla^{2} T+Q_{c}(1-\epsilon) \rho_{c} A_{c} \exp \left[\frac{-E_{c}}{R T}\right] \mathcal{O}_{2} \\
\quad+Q_{b}(1-\epsilon) \rho_{b} \rho_{c} \frac{A_{1} \exp \left[\frac{-E_{1}}{R T}\right]}{1+A_{2} \exp \left[\frac{-E_{2}}{R T}\right]}
\end{array}
\end{aligned}
$$

Oxygen $\left(\mathcal{O}_{2}\right)$ for $x \in[0, L]$ and $y \in[0, h]$

$$
\epsilon \frac{\partial \mathcal{O}_{2}}{\partial t}=D_{o, \text { eff }} \nabla^{2} \mathcal{O}_{2}-A_{c}(1-\epsilon) \rho_{c} \exp \left[\frac{-E_{c}}{R T}\right] \mathcal{O}_{2},
$$

Algebraic relationships

$$
\begin{aligned}
& k_{\text {eff }}=\epsilon k_{\text {air }}+(1-\epsilon) k_{\mathrm{c}}, \\
& (\rho C)_{\text {eff }}=\epsilon \rho_{\text {air }} C_{\text {air }}+(1-\epsilon) \epsilon \rho_{c} C_{c}, \\
& D_{o, \text { eff }}=\epsilon D_{o, \text { air }} .
\end{aligned}
$$

The terms that appear in equations (1)-(5) are defined in the nomenclature (see Section 5 ). Here, $k_{\text {eff }}$ and $D_{o \text {,eff }}$ are effective properties which are considered to be independent of temperature and concentration. The system above describes the process of heat conduction and diffusion of oxygen in the standard way. The heat produced due to oxidation of the cellulosic material is represented by the second term on the right-hand side of the energy equation (1), which also appear in equation (2) describing the concentration of oxygen within the pile. However, unlike previous investigations into spontaneous ignition in bulk solids, we include the heat generated due to the biological activity within the pile - represented by the third term in equation (1). In this term, the growth parameters $A_{1}$ and $E_{1}$ reflect that at low temperatures the metabolic activity of the biomass grows with increasing temperature as enzyme activity increases. However, for sufficiently high 
temperatures the essential proteins which are sensitive to heat begin to denature leading to cell death. These processes are represented by the biomass deactivation parameters $A_{2}$ and $E_{2}$. To ensure that the heat release due to the biological activity within the compost pile has a global maximum in the temperature range, the activation energy for the inhibition process must be larger than the activation energy for the biomass growth, that is, $E_{2}>E_{1}$. The detailed formulation of the term representing the heat generated by the biomass is obtained from Chen \& Mitchell [3].

The algebraic expressions (3)-(5) give the relationships for the effective thermal conductivity, the effective thermal capacity of the compost pile in terms of corresponding properties of air and pure cellulosic material, and the effective diffusion coefficient for oxygen.

Since this is a preliminary investigation, we assume only simple boundary conditions for the system. That is, we assume that the surface in contact with air around the whole slab has ambient conditions for both the temperature and oxygen (Dirichlet type): $T=T_{a}$ and $\mathcal{O}_{2}=\mathcal{O}_{2, a}$ along $x=0, x=L$ and $y=h$. As for the base of the pile in contact with the ground $(y=0)$, we assume a Neumann condition for the oxygen and a Dirichlet condition for the temperature. In other words, we assume that the ground is impermeable to oxygen $\left(\partial \mathcal{O}_{2} / \partial n=0\right.$, where $\partial / \partial n$ describes the outward normal derivative at the boundary), whereas we assume that the temperature at the base of the pile is a perfect conductor $\left(T=T_{g}\right)$. Sexton et al. [13] stated that such conditions for the base of the pile appears to closely approximate the conditions currently found for bagasse piles. We also assume that the initial temperature and oxygen distribution within the pile are the same as the ambient conditions. 


\section{$3 \quad$ Results}

For the one-dimensional investigation we used the continuation and bifurcation software Auto97 [4] to determine the steady state solutions. Note that Auto does not determine stability for boundary value problems; however, by solving the time dependent version of the problem we ascertained the stability of the solution branches. The solutions from our two-dimensional rectangular slab investigation were obtained using a time and space adaptive finite element package FLEXPDE [6] . Due to its adaptive nature, errors in both space and time are minimized as mesh points are added in regions of large gradients particularly on the surface of the compost pile. All calculations were performed to a relative error tolerance of less than $0.1 \%$.

\subsection{One-dimensional case}

Figure 1 shows the results from our one-dimensional investigation. The results from the two models (with and without oxygen consumption) have excellent agreement up to $L=28.8 \mathrm{~m}$. As the compost length is increased from $L=0$, the temperature within the pile also increases very slowly along the lowest solution branch, sometimes referred to as the no-reaction branch. There is a fold (or limit) point at approximately $L=16.8 \mathrm{~m}$, when the solution branch commences to "fold back". We refer to this limit point as the low temperature ignition point. All solutions along this lowest solution branch $0<L<16.8 \mathrm{~m}$ were found to be stable. There exists an intermediate solution branch between $9.5 \mathrm{~m}<L<16.8 \mathrm{~m}$ which was found to be unstable. At $L=9.5 \mathrm{~m}$, there exists another limit point, often referred to as the extinction limit point. The solution branch changes stability at this limit point from the unstable intermediate temperature branch to the stable elevated temperature branch. Hence there is a region of bi-stability between the low temperature ignition limit point and the extinction limit point. For the model that includes oxygen consumption (bold red curve), the solution 


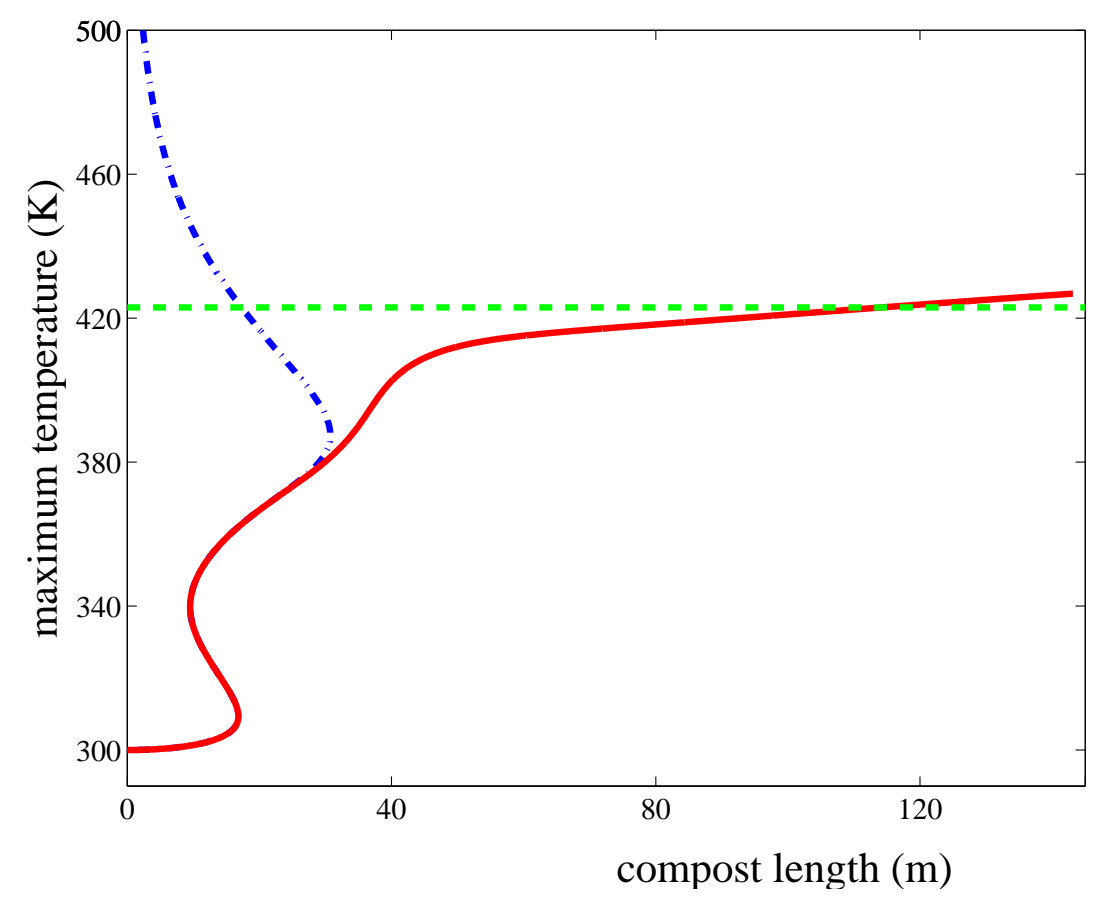

Figure 1: The dependence of maximum temperature within the onedimensional compost heap upon the pile length. The red (bold) and blue (dot-dashed) curves represent results obtained from models that included and excluded oxygen consumption respectively. The horizontal dashed green line at temperature $423^{\circ} \mathrm{K}$ represents typical ignition temperature for compost materials. 
remains stable beyond this extinction limit point. However, for the model which neglects oxygen consumption (blue dot-dashed curve), there exists yet another limit point at $L=30.7 \mathrm{~m}$, which we refer to as the high temperature ignition point. Once again the solution branch for this model changes stability at this fold point. From an operational viewpoint, the elevated temperature branch may be desirable since composting is enhanced at such temperatures.

For compost lengths slightly larger than the value at the low temperature ignition point $(L=16.6 \mathrm{~m})$, the maximum steady state temperature converges onto the elevated solution branch. For example, if $L=17.5 \mathrm{~m}$ the maximum steady state temperature within the one-dimensional pile is approximately $363^{\circ} \mathrm{K}$ (predicted by both models). However, if we let $L=31 \mathrm{~m}$, beyond the high temperature limit point for the model corresponding to no oxygen consumption, then we obtain different predictions - ignition within the pile for the model with no oxygen consumption, whereas a maximum steady state temperature of approximately $382^{\circ} \mathrm{K}$ within the pile for the model with oxygen consumption. Ignition for the model with oxygen consumption only occurs for very long (or very wide) piles, that is for piles with lengths greater than $114 \mathrm{~m}$. Here we note that Rynk [12] stated that compost materials generally ignite at $423^{\circ} \mathrm{K}\left(150^{\circ} \mathrm{C}\right)$, and this is denoted by the horizontal dashed green line in Figure 1. Piles over a hundred metres long (or wide) may be more relevant to the large dumps of urban waste near any of the large cities around the world.

Before we commenced our investigation of the two-dimensional case, we used the finite element package FLEXPDE to obtain solutions to the onedimensional problem described above, as a check of its accuracy. We found that the results from both packages were accurate up to four decimal places. 


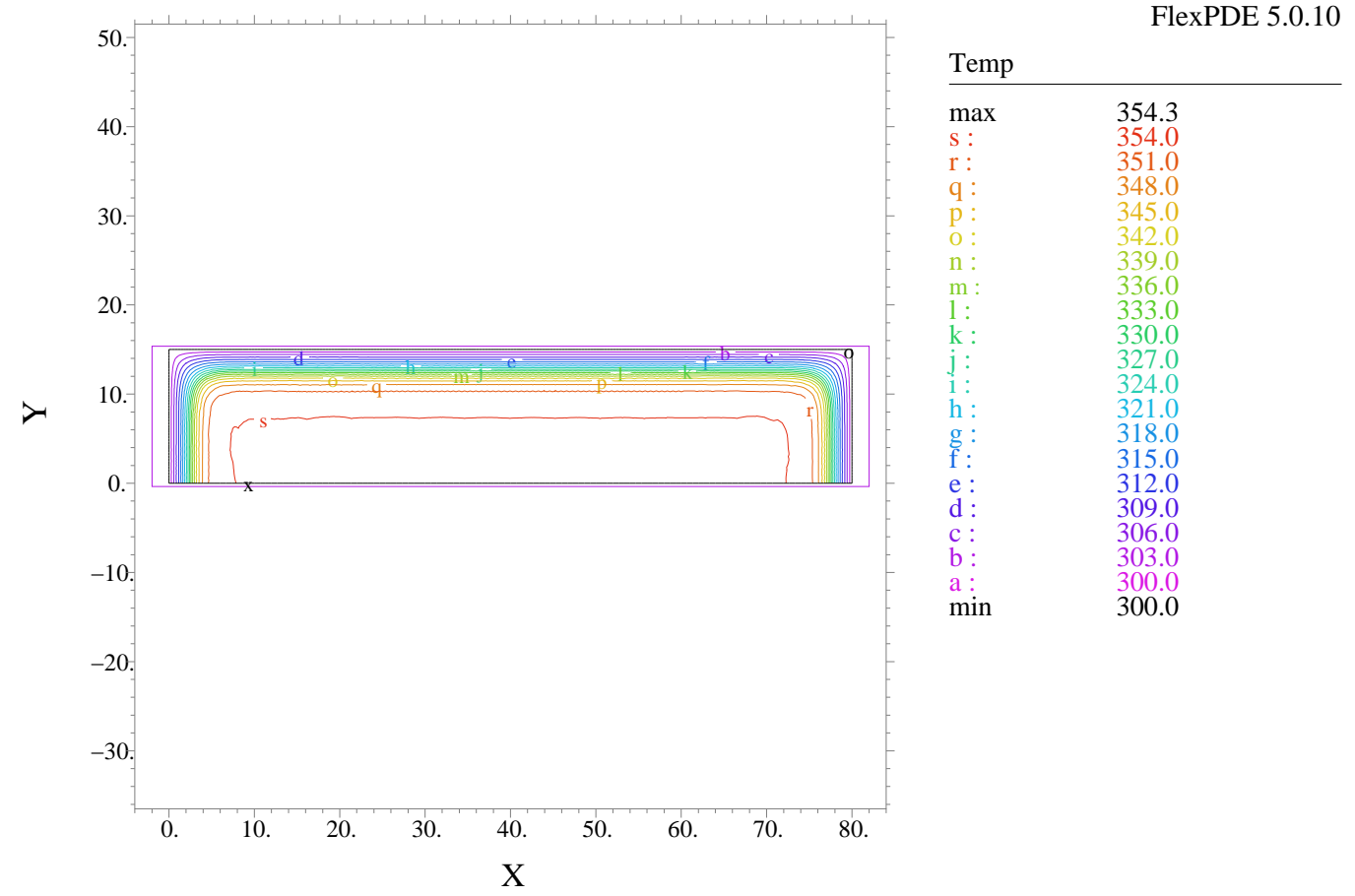

FiguRE 2: The output from FLEXPDE showing the temperature distribution (for the model with oxygen consumption) within the compost pile after 180 days. The rectangular pile has a length of $80 \mathrm{~m}$ and a height of $18 \mathrm{~m}$. 


\subsection{Two-dimensional case}

Here we fix the length of the pile to be $80 \mathrm{~m}$ and examine the effects of varying the height of the rectangular slab. The length of the pile is chosen arbitrarily as an illustration of the behaviour of the model; however, note that industrial compost piles can be over one hundred metres in length. Figure 2 is the output from FLEXPDE showing the temperature distribution within the pile after 180 days for the case of a rectangular slab $18 \mathrm{~m}$ high. These results correspond to the model that includes oxygen consumption. The maximum temperature of $354.3^{\circ} \mathrm{K}$ is located in the middle of the pile, and the minimum temperature of $300^{\circ} \mathrm{K}$ occurs at the compost-air (and compost-ground) surface. The steady state temperature of this compost pile is around $380^{\circ} \mathrm{K}$.

Figure 3 shows the stable maximum steady state temperature plot for the case when the height of the pile is varied. This figure shows that the solutions for the two models (with and without oxygen consumption) agree along the lowest solution branch (the no-reaction branch), and the lower portion of the elevated solution branch. The discrepancy between the models arises when the model with no oxygen consumption predicts a high temperature ignition limit point at around $h=15.7 \mathrm{~m}$, whereas the model including oxygen consumption does not possess such a limit point. Consequently the maximum steady state temperature gradually rises as the height of the slab is increased. Similar behaviour between the two models was observed in the one-dimensional investigation when the length of the pile was increased (Figure 1).

\section{Conclusions}

We undertook a preliminary investigation of a one- and two-dimensional, spatially dependent model for the self-heating of a compost heap. This model incorporates terms that account for both the biological and chemical ac- 


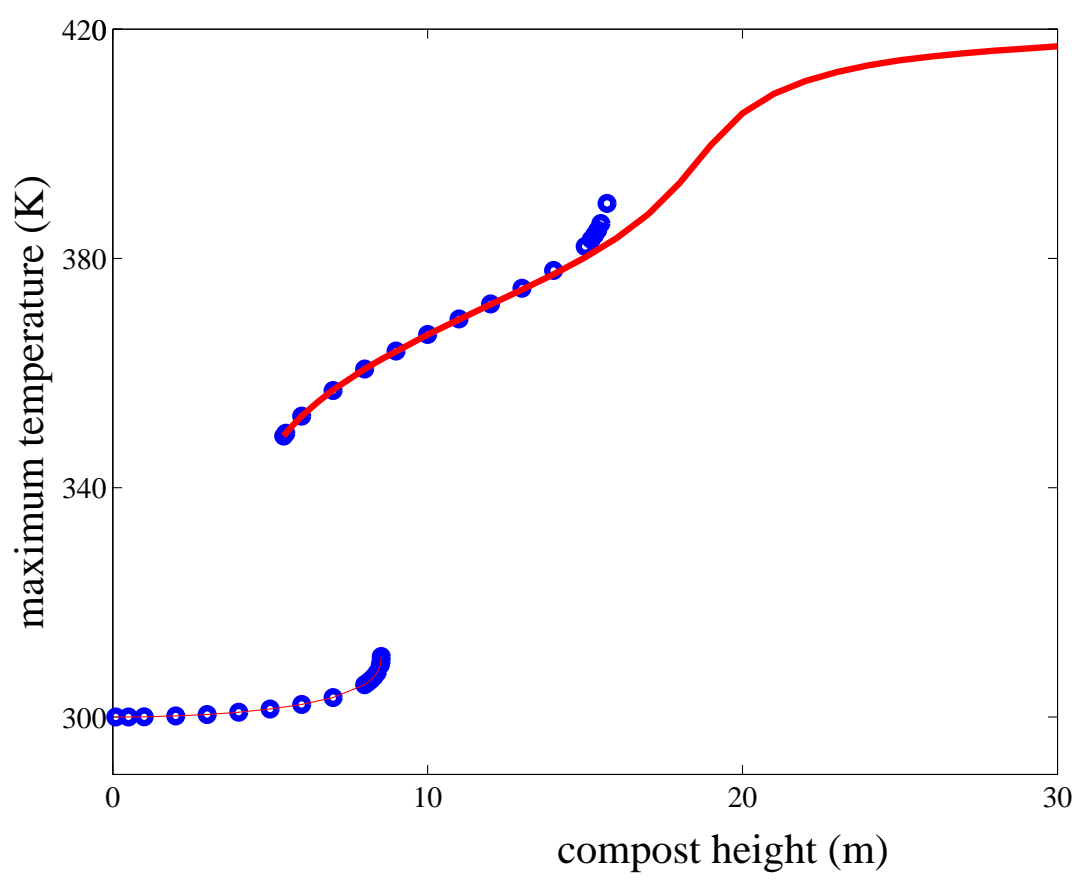

Figure 3: The dependence of maximum temperature within the compost heap upon the height of the pile. The length of the compost pile is fixed at $80 \mathrm{~m}$. The stable steady state solution branch represented by the red curve corresponds to the solutions obtained from the model with oxygen consumption, whereas the solutions represented by the blue circles were obtained from the model with no oxygen consumption. 
tivities which are known to occur within the compost heap, as well as the consumption of oxygen. We believe that the work investigated here forms the most realistic model of composting so far. For simplicity we assumed the geometry of the compost heap to be a two-dimensional rectangular slab.

The model exhibits two stable low temperature branches. The lowest of these represents a state of negligible heat reaction which is undesirable for composting purposes. The higher branch, on the other hand, is an elevated temperature branch, which is the feature of interest in industrial composting facilities and municipal tips. Although highly simplified, the current model predicts the features of interest.

Comparing the two models, with and without oxygen consumption in the one- and two-dimensional cases, we found that the model without oxygen consumption seems to always predict a high temperature ignition limit point on the elevated temperature solution branch, beyond which flaming combustion occurs within the pile. However, this feature is absent for the model that considers oxygen consumption, and the temperature gradually rises as the dimensions of the pile is increased. This difference between the two systems is expected since the consumption of oxygen would reduce the rate of heat production within the pile, lowering the temperature. This scenario would change if there is a steady supply of oxygen into the pile.

\section{$5 \quad$ Nomenclature and typical parameter values}

$A_{C}$ Pre-exponential factor for the oxidation of the cellulosic material $(1.8 \times$ $\left.10^{4} \mathrm{~s}^{-1}\right)$

$A_{1}$ Pre-exponential factor for the oxidation of the biomass growth $(2.0 \times$ $\left.10^{6} \mathrm{~s}^{-1}\right)$ 
$A_{2}$ Pre-exponential factor for the inhibition of biomass growth $\left(6.86 \times 10^{30}\right)$

$C_{\text {air }}$ Heat capacity of air $\left(1005 \mathrm{~J} \mathrm{~kg}^{-1} \mathrm{~K}^{-1}\right)$

$C_{c}$ Heat capacity of the cellulosic material $\left(3320 \mathrm{~J} \mathrm{~kg}^{-1} \mathrm{~K}^{-1}\right)$

$E_{c}$ Activation energy for the oxidation of the cellulosic material $(110 \times$ $10^{3} \mathrm{~J} \mathrm{~mol}^{-1}$ )

$E_{1}$ Activation energy for the biomass growth $\left(100 \times 10^{3} \mathrm{~J} \mathrm{~mol} \mathrm{biomass}^{-1}\right)$

$E_{2}$ Activation energy for inhibition of biomass growth $\left(200 \times 10^{3} \mathrm{~J} \mathrm{~mol} \mathrm{biomass}^{-1}\right.$

$L$ Length of the compost pile $(\mathrm{m})$

$\mathcal{O}_{2}$ Oxygen concentration within the pile $\left(\mathrm{kg} \mathrm{m}^{-3}\right)$

$\mathcal{O}_{2, a}$ Ambient oxygen concentration $\left(0.272 \mathrm{~kg} \mathrm{~m}^{-3}\right)$

$Q_{b}$ Exothermicity for the oxidation of biomass per $\mathrm{kg}$ of dry cellulose $(6.66 \times$ $10^{6} \mathrm{~J} \mathrm{~kg}^{-1}$ )

$Q_{c}$ Exothermicity for the oxidation of the cellulosic material $\left(1.7 \times 10^{7} \mathrm{~J} \mathrm{~kg}^{-1}\right)$

$R$ Ideal gas constant $\left(8.314 \mathrm{~J} \mathrm{~K}^{-1} \mathrm{~mol}^{-1}\right)$

$T$ Temperature within the compost pile (K)

$T_{a}$ Ambient temperature $(300 \mathrm{~K})$

$T_{g}$ Temperature of the ground $(300 \mathrm{~K})$

$h$ Height of the compost pile (m)

$k_{\text {air }}$ Effective thermal conductivity of air $\left(0.026 \mathrm{~W} \mathrm{~m}^{-1} \mathrm{~K}^{-1}\right)$

$k_{c}$ Effective thermal conductivity of cellulose $\left(0.3 \mathrm{~W} \mathrm{~m}^{-1} \mathrm{~K}^{-1}\right)$

$k_{\text {eff }}$ Effective thermal conductivity of the bed $\left(\mathrm{W} \mathrm{m}^{-1} \mathrm{~K}^{-1}\right)$ 
$t$ Time (s)

$x$ Spatial distance along the length of the pile $(\mathrm{m})$

y Spatial distance along the width of the pile $(\mathrm{m})$

$\epsilon$ Void fraction $(0.3)$

$(\rho C)_{\text {eff }}$ Effective thermal capacity per unit volume of the bed $\left(\mathrm{J} \mathrm{m}^{-3} \mathrm{~K}^{-1}\right)$

$\rho_{\text {air }}$ Density of air $\left(1.17 \mathrm{~kg} \mathrm{~m}^{-3}\right)$

$\rho_{b}$ Density of bulk biomass within the compost pile $\left(575 \mathrm{~kg} \mathrm{~m}^{-3}\right)$

$\rho_{c}$ Density of pure cellulosic material $\left(1150 \mathrm{~kg} \mathrm{~m}^{-3}\right)$.

\section{References}

[1] P. C. Bowes. Self-heating: evaluating and controlling the hazard. Elsevier, Amsterdam, 1984. C136, C137

[2] W. F. Brinton Jr., E. Evans, M. L. Droffner and R. B. Brinton, Standartized test for evaluation of compost self-heating, BioCycle, 36, 1995, 65-60. C136

[3] X. D. Chen and D. A. Mitchell. Start-up strategise for self-heating and efficient growth in stirred bioreactors for solid state fermentation. Proceedings of the 24th Annual Australian and New Zealand Chemical Engineering Conference (CHEMECA 96), 4:111-116, 1996. C139

[4] E. J. Doedel, T. F. Fairgrieve, B. Sandstede, A. R. Champneys, Y. A. Kuznetsov and X. Wang AUTO 97: Continuation and bifurcation software for Ordinary Differential Equations (with HomCont). ftp://ftp.cs.concordia.ca/pub/doedel/auto. C140 
[5] D. A. Frank-Kamenetskii. Diffusion and heat transfer in chemical kinetics. Plenum Press, New York (Second Edition), 1969. C136, C137

[6] FLEXPDE. PDE Solutions, Inc. http://www.pdesolutions .com. C140

[7] M. Golubitsky and D. Schaeffer. The classification theorem in singularities and groups in Bifurcation theory (First edition) Springer, Berlin, 1985. C137

[8] W. Hogland, T. Bramryd and I. Persson, Physical, biological and chemical effects of unsorted fractions of industrial solid waste in waste fuel storage, Waste Management and Research, 14, 1996, 197-210. C136

[9] P. F. Hudak, Spontaneous combustion of shale spoils at sanitary landfill, Waste Management, 22, 2001, 687-688. C137

[10] M. I. Nelson, E. Balakrishnan and X. D. Chen, A Semenov model of self-heating in compost piles, Transactions of IChemE, B81, 2003, 375-383. C137

[11] D. Nield and A. Bejan. Convection in porous media, Springer-Verlag, New york, 1992. C137

[12] R. Rynk, Fires at composting facilities, BioCycle Magazine, 41, 2000, 54-58. C136, C142

[13] M. J. Sexton, C. Macaskill and B. F. Gray, Self-heating and drying in two-dimensional bagasse piles, Combust. Theory and Model., 5, 2001, 517-536. C139

[14] H. S. Sidhu, M. I. Nelson, N. A. Ansari and X. D. Chen, Mathematical analysis of self-heating in compost piles. Proceedings of the 34th Annual Australasian Chemical Engineering Conference (CHEMECA 2006), ISBN 0-86869-110-0. C137 


\section{Author addresses}

1. H. S. Sidhu, School of Physical, Environmental and Mathematical Sciences, University of New South Wales at the Defence Force Academy, Australia.

mailto:h.sidhu@adfa.edu.au

2. M. I. Nelson, School of Mathematics and Applied Statistics, The University of Wollongong

3. X. D. Chen, Department of Chemical Engineering, Monash University 